Article

\title{
Doubly-Excited States of Beryllium-Like Ions with Screened Coulomb Potentials
}

\author{
Arijit Ghoshal ${ }^{1, *(D)}$ and Yew Kam Ho ${ }^{2}$ \\ 1 Department of Mathematics, Burdwan University, Golapbag, Burdwan 713104, West Bengal, India \\ 2 Institute of Atomic and Molecular Sciences, Academia Sinica, P.O. Box 23-166, Taipei 106, Taiwan; \\ ykho@pub.iams.sinica.edu.tw \\ * Correspondence: arijit98@yahoo.com; Tel.: +91-94341-07364
}

Received: 6 July 2018; Accepted: 30 July 2018; Published: 1 August 2018

\begin{abstract}
The screening effects of the interaction potentials on the lowest ${ }^{1} S$ doubly-excited states of beryllium-like ions were investigated by calculating the density of resonance states within the framework of the stabilization method. Two types of screened interaction potentials, namely static screened Coulomb potential and exponential cosine screened Coulomb potential, were taken into consideration. A model potential was used to describe the interaction between the core and outer electrons, and the Be-like ions were treated as being effectively three-body systems. Calculations were performed for Be and $B^{+}$. It was possible to calculate the energy and width of one doubly-excited state of Be and four doubly-excited states of $B^{+}$lying above the $1 s^{2} 2 p$ threshold. Significant changes were found to exist in the behaviour of the width with varying screening parameters. To the best of our knowledge, such an investigation on the doubly-excited states of Be-like ions under screened environments is the first reported calculation of this type in the literature.
\end{abstract}

Keywords: beryllium-like ions; screened potentials; doubly-excited states; resonance; stabilization method

\section{Introduction}

The effects of screened Coulomb potentials (SCP) on the structural and collisional properties of atomic systems have long been subjects of extensive study [1-11]. The Coulomb field of potential around a charge often gets modified or screened due to the influence of an external filed. In few cases, closed-form expressions of screened potentials can be obtained by solving the corresponding Poisson equation. For example, the field of potential around a test charge embedded in weakly coupled plasmas can be adequately described by the static screened Coulomb potential (SSCP) of the following form [1]:

$$
V(r)=\frac{1}{r} e^{-\lambda r} \quad \text { (in a.u.), }
$$

where $\lambda$ is a function of plasma parameters, known as the screening parameter, and SSCP is a well-known potential. Apart from its occurrence in plasma physics, it occurs in various branches of physics. Another SCP of this kind is the exponential cosine screened Coulomb potential (ECSCP) of the form:

$$
V(r)=\frac{1}{r} e^{-\lambda r} \cos (\lambda r) \quad \text { (in a.u.) }
$$

where $\lambda$ is the screening parameter. In plasma physics, this type of potential is used to describe the potential around a test charge embedded in quantum plasmas [12]. Besides plasma physics, ECSCPs play fundamental roles in various other branches of physics [13]. Apart from the above-mentioned screened potentials, a number of screened potentials exist in the literature, such as the Hulthen potential, [14]. It has to be mentioned that the screening parameter $(\lambda)$, which appears in Equations (1) and (2), carries 
different information for different physical situations. Both the potentials (1) and (2) reduce to the pure Coulomb potential when $\lambda=0$. Furthermore, for a given value of $\lambda$, SSCP is stronger than ECSCP. When the interactions among the charged particles in an atomic system are governed by SCP, the structural properties of the system get significantly modified in contrast to the free-atomic system. It is therefore pertinent to look into the changes that an atomic system may undergo when it is subjected to SCP.

In this paper, we make an attempt to study the lowest ${ }^{1} S$ doubly-excited states (DES) of Be-like ions in which interactions among the charged particles are governed by SCP of the types given in Equations (1) and (2). The DESs of an atomic system play a fundamental role in the corresponding electron-ion scattering. The decay of such states gives rise to complex resonance structures in the scattering cross section. Knowledge about these resonances is an important tool for getting information about the structure of complex atomic systems. Be-like ions are complex four-electron atomic systems. The structures and spectra of their DESs provide us fruitful information to understand the complicated electron correlation effects, corresponding electron-ion scattering processes, as well as plasma diagnostics. A number of investigations have been made so far to study different DESs in Be-like ions in a vacuum, both theoretically and experimentally [15-38]. However, the behaviour of the DESs of Be-like ions under a screening environment is yet to be investigated. Performing calculations for multi-electron atoms or ions is a cumbersome task and, in fact, poses enormous computational difficulties due to the involvement of the number of coordinates that is equal to the number of electrons. In this work, we circumvent the involvement of many electrons by resorting to the 'method of model potential' (MMP) which gives us the opportunity to consider a multi-electron atom/ion as consisting of a frozen positive core together with valence (active) electrons going around the frozen core. The frozen core includes the nucleus as well as the other (passive) electrons. Taking into account the effects of passive electrons, the interaction between the frozen core and an active electron is chosen (or modeled) so that the binding energies of the residual system coincide with the observed data [39]. The interaction potential between the frozen core and an active electron is called the model potential (MP). In the present paper, by following the MMP, we consider a Be-like ion to be a two-electron system consisting of a frozen core (nucleus plus $1 s^{2}$ electrons) and two $2 \mathrm{~s}$ active electrons. The interaction between the core and an active electron is then modelled by a suitable model potential. Within this framework, we apply the stabilization method (SM) to calculate the energies and widths of the DESs of Be-like ions. Here, we restrict our calculations to $Z=4$ and $Z=5$ only, where $Z$ denotes the atomic number, that is $B e$ and $B^{+}$. Atomic units (a.u.) are used throughout the remaining part of this manuscript unless stated otherwise.

\section{Method and Calculations}

Let $\vec{r}_{1}$ and $\vec{r}_{2}$ be the coordinates of the active electrons relative to the core (assumed to be at rest), and let $\vec{r}_{12}\left(=\vec{r}_{1}-\vec{r}_{2}\right)$ be their relative coordinate. The method of model potential has been extensively discussed in the literature [39-42], and hence we do not need to discuss it here again. In order to describe the electrostatic interaction between the core and an active electron, Bachau et al. [28] proposed a model potential of the form (in a.u.)

$$
V_{M}\left(\vec{r}_{1}\right)=-\frac{1}{r_{1}}\left[(Z-2)+2\left(1+\alpha r_{1}\right) e^{-2 \alpha r_{1}}\right],
$$

where $\alpha$ is the effective charge felt by the two electrons described by the $1 s$ orbitals. In a screening environment, such as that given by Equations (1) and (2), the above potential takes the form

$$
V_{M}\left(\vec{r}_{1}\right)=-\frac{e^{-\lambda r_{1}}}{r_{1}}\left[(Z-2)+2\left(1+\alpha r_{1}\right) e^{-2 \alpha r_{1}}\right] \cos \left(\epsilon \lambda r_{1}\right)
$$


where $\epsilon=0$ for SSCP and $\epsilon=1$ for ECSCP respectively. With this choice of MPs, the non-relativistic Hamiltonian of Be-like ions interacting with SSCP/ECSCP is given by

$$
H=-\frac{1}{2} \nabla_{1}^{2}-\frac{1}{2} \nabla_{2}^{2}+V_{M}\left(\vec{r}_{1}\right)+V_{M}\left(\vec{r}_{2}\right)+\frac{e^{-\lambda r_{12}}}{r_{12}} \cos \left(\epsilon \lambda r_{12}\right) .
$$

For the above Hamiltonian, the spectral density $(\rho(E))$ is calculated with $H \Psi=E \Psi$ by employing the stabilization method. Ho and co-workers [43-47] used this method to determine the resonance parameters (energy, $E_{r}$ and width, $\Gamma$ ) of atomic systems; hence, here, we just give an outline of the method instead of presenting the derivation of the method in detail. In the Feshbach projection formalism, $\rho(E)$ is the union of $\rho^{P}(E)$ and $\rho^{Q}(E)$, where $P$ and $Q$ respectively stand for the open space and closed space [47]. $\rho^{P}(E)$ is a smooth function of the energy $(E)$, whereas $\rho^{Q}(E)$ emerges as a consequence of the complex poles of the underlying Green's function, and it is related to the isolated resonances. For an isolated resonance, it can be shown that $\rho^{Q}(E)$ assumes the following Lorentzian form [48]:

$$
\rho^{Q}(E) \cong x_{0}+\frac{A}{\pi} \frac{\Gamma / 2}{\left(E-E_{r}\right)^{2}+(\Gamma / 2)^{2}},
$$

where $x_{0}$ is the baseline offset, $A$ is the total area under the curve from the base line, $E_{r}$ is the centre of the peak, and $\Gamma$ denotes the full width of the peak of the curve at half height (as shown in Figure 1).

In this work, we use the following wave function to calculate $\rho^{Q}(E)$ :

$$
\begin{gathered}
\Psi\left(\vec{r}_{1}, \vec{r}_{2}\right)=\sum_{i=1}^{N} C_{i} \psi_{i}=\sum_{l_{i}+m_{i}+n_{i}=0}^{\omega} C_{i}(1+\hat{P}) e^{-s\left(r_{1}+r_{2}\right)} r_{1}^{l_{i}} r_{2}^{m_{i}} r_{12}^{n_{i}} \\
l_{i}, m_{i}, n_{i}=0,1,2, \cdots, l_{i} \geq m_{i}
\end{gathered}
$$

where $C_{i}(i=1,2,3, \cdots, N)$ are linear expansion coefficients, $0<s<1$ is a scaling parameter, and $\hat{P}$ is the exchange operator such that $\hat{P} f\left(\vec{r}_{1}, \vec{r}_{2}\right)=f\left(\vec{r}_{2}, \vec{r}_{1}\right)$ for arbitrary function $f$. The number of terms $(N)$ in the above wavefunction is determined by $\omega$ in such a way that $\omega=0$ stands for $N=1 ; \omega=1$ stands for $N=4$; and so on. For a given value of the screening parameter $(\lambda)$, we employed the wave function (7) to obtain the energy levels of $H$ as a function of the scaling parameter (s), which led to the stabilization diagram $E(s)$ (as shown in Figure 2). The density of the resonance state for the n-th energy level is given by

$$
\rho_{n}^{Q}(E)=\left[\frac{E_{n}\left(s_{i+1}\right)-E_{n}\left(s_{i-1}\right)}{s_{i+1}-s_{i-1}}\right]_{E_{n}\left(s_{i}\right)=E}^{-1} .
$$

In general, $E$ varies slowly with $s$, and hence, $\rho_{n}^{Q}(E)$. However, $E$ changes rapidly with $s$ in the neighbourhood of points at which $E(s)$ takes a sharp turn, causing $\rho_{n}^{Q}(E)$ to take the form of a Lorentzian profile similar to Equation (6). These points are called the points of avoided crossing (as shown in Figure 2). We calculated $\rho_{n}^{Q}(E)$ from each pair of consecutive energy levels in the neighbourhood of each avoided crossing. $\rho_{n}^{Q}(E)$ was then fitted with Lorentzian form (6) to get the resonance energy $\left(E_{r}\right)$ and resonance width $(\Gamma)$. The resonance parameters for that particular resonance were extracted from the best fitting (where the value of the "coefficient of determination" $R^{2}$ is closest to unity). 

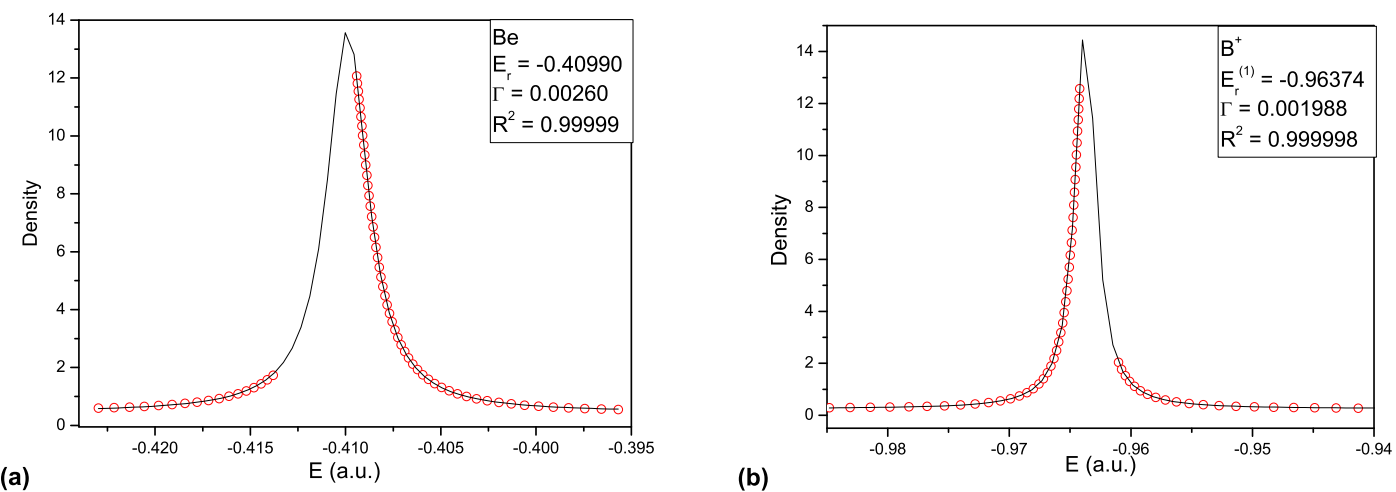

Figure 1. The density of resonance states fitted to the Lorentzian form corresponding to the lowest resonance, as indicated in Figures 2a,b, respectively. The circles are the calculated values and the solid line is the fit function. The resonance parameters are shown in the box in the top right corner. (a) Be, (b) $B^{+}$.

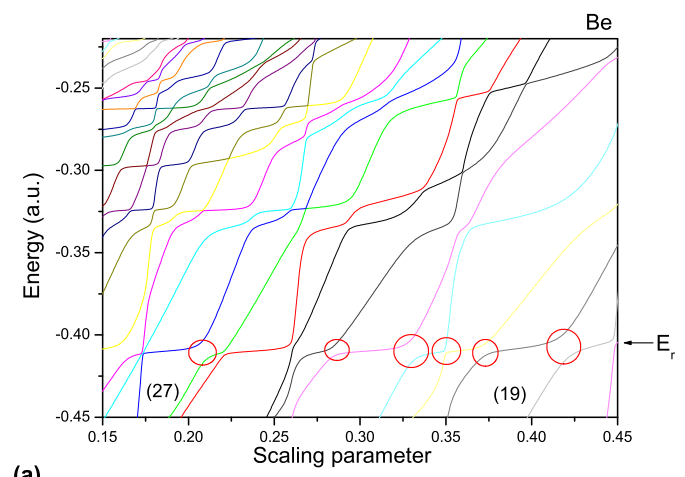

(a)

Figure 2. Stabilization diagram for $\lambda=0.0$ obtained by considering $N=372$ and giving $s$ an increment of 0.0005 units in the wave function (7). The numbers in parenthesis left of the solid line show the order of appearance of the energy levels. The arrow indicates the position of resonance, and the circles indicate the points of avoided crossing. (a) $\mathrm{Be},(\mathbf{b}) \mathrm{B}^{+}$.

\section{Results and Discussion}

First, we examined the effectiveness of the model potential (4) used to represent the interaction between the core and an active electron in Be-like ions. We determined the binding energies of $B e^{+}$ and $B^{++}$within the framework of Ritz's variational principle by employing a radial wave function of the form

$$
R(r)=\sum_{i}^{N_{1}} D_{i} e^{-a_{i} r} r_{i}, \quad l_{i}=0,1,2, \cdots,
$$

where $D_{i}$ is the expansion coefficient, and $a_{i}$ is a nonlinear variational parameter. We used $\alpha=Z-5 / 16$ in the model potential (3), as mentioned by Bachau et al. [28]. It was found that a convergent result of up to 10 decimal places can be obtained for binding energies by considering $N_{1}=30$ in the above wave function. The binding energies of $B e^{+}$and $B^{++}$thus obtained along with the experimental data [49] are presented in Table 1 . From Table 1, it can be seen that our present results for the unscreened case are in agreement with the experimental observations [49]. 
Table 1. Energies (-E a.u.) of the $1 s^{2} n l$ states of $B e^{+}$and $B^{++}$ions in static screened Coulomb potential (SSCP) and exponential cosine screened Coulomb potential (ECSCP) determined by using the wave function (9). All values refer to the respective core energies. A and B respectively stand for SSCP and ECSCP. Numbers with superscripts ' $a$ ', ' $b$ ' and ' $c$ ' denote the corresponding results of Reference [49] (experimental observation) and Reference [50,51] respectively.

\begin{tabular}{|c|c|c|c|c|c|}
\hline \multirow{2}{*}{$\lambda\left(\right.$ in $\left.a_{0}^{-1}\right)$} & \multirow{2}{*}{$n l$} & \multicolumn{2}{|c|}{$B e^{+}$} & \multicolumn{2}{|c|}{$\overline{B^{++}}$} \\
\hline & & A & B & A & B \\
\hline \multirow{17}{*}{0.0} & \multirow[t]{3}{*}{$2 s$} & 0.66964102 & 0.66964102 & 1.39474181 & 1.39474181 \\
\hline & & $0.669246^{a}$ & $0.669246^{a}$ & $1.3936211^{b}$ & $1.3936211^{b}$ \\
\hline & & & & $1.394522^{c}$ & $1.394522^{c}$ \\
\hline & \multirow[t]{2}{*}{$2 p$} & 0.52430329 & 0.52430329 & \multirow[t]{2}{*}{1.17584781} & \multirow[t]{2}{*}{1.17584781} \\
\hline & & $0.523768^{a}$ & $0.523768^{a}$ & & \\
\hline & \multirow[t]{2}{*}{$3 s$} & 0.26775134 & 0.26775134 & \multirow[t]{2}{*}{0.57384421} & \multirow[t]{2}{*}{0.57384421} \\
\hline & & $0.267233^{a}$ & $0.267233^{a}$ & & \\
\hline & \multirow[t]{2}{*}{$3 p$} & 0.22998134 & 0.22998134 & \multirow[t]{2}{*}{0.51587898} & \multirow[t]{2}{*}{0.51587898} \\
\hline & & $0.229582^{a}$ & $0.229582^{a}$ & & \\
\hline & \multirow[t]{2}{*}{$3 d$} & 0.22240325 & 0.22240325 & \multirow[t]{2}{*}{0.50052384} & \multirow[t]{2}{*}{0.50052384} \\
\hline & & $0.222478^{a}$ & $0.222478^{a}$ & & \\
\hline & \multirow[t]{2}{*}{$4 \mathrm{~s}$} & 0.14340301 & 0.14340301 & \multirow[t]{2}{*}{0.31137200} & \multirow[t]{2}{*}{0.31137200} \\
\hline & & $0.143153^{a}$ & $0.143153^{a}$ & & \\
\hline & \multirow[t]{2}{*}{$4 \mathrm{p}$} & 0.12833340 & 0.12833340 & \multirow[t]{2}{*}{0.28803005} & \multirow[t]{2}{*}{0.28803005} \\
\hline & & $0.128134^{a}$ & $0.128134^{a}$ & & \\
\hline & \multirow[t]{2}{*}{$4 \mathrm{~d}$} & 0.12510394 & 0.12510394 & 0.28154683 & 0.28154683 \\
\hline & & $0.125124^{a}$ & $0.125124^{a}$ & & \\
\hline & $2 s$ & 0.62984556 & 0.62898469 & 1.33486968 & 1.33392802 \\
\hline & $2 p$ & 0.48484262 & 0.48395393 & 1.11620861 & 1.11529802 \\
\hline & $3 s$ & 0.22974020 & 0.22776016 & 0.51592131 & 0.51376335 \\
\hline & $3 p$ & 0.19217000 & 0.19007265 & 0.45805733 & 0.45585483 \\
\hline 0.02 & $3 d$ & 0.18441982 & 0.18255588 & 0.44255868 & 0.44062172 \\
\hline & $4 \mathrm{~s}$ & 0.10723572 & 0.10391841 & 0.25541166 & 0.25171891 \\
\hline & $4 p$ & 0.09242998 & 0.08894775 & 0.23221675 & 0.22842460 \\
\hline & $4 d$ & 0.08900850 & 0.08569234 & 0.22553449 & 0.22195201 \\
\hline & $2 s$ & 0.57337057 & 0.56838958 & 1.24858165 & 1.24301496 \\
\hline & $2 p$ & 0.42898087 & 0.42386046 & 1.03015901 & 1.02477217 \\
\hline & $3 s$ & 0.18011693 & 0.16985931 & 0.43708142 & 0.42525691 \\
\hline & $3 p$ & 0.14331425 & 0.13260204 & 0.37954810 & 0.36752946 \\
\hline 0.05 & $3 d$ & 0.13444727 & 0.12462687 & 0.36285344 & 0.35206529 \\
\hline & $4 \mathrm{~s}$ & 0.06551481 & 0.05095356 & 0.18528180 & 0.16713958 \\
\hline & $4 p$ & 0.05183304 & 0.03695721 & 0.16272235 & 0.14425590 \\
\hline & $4 \mathrm{~d}$ & 0.04755823 & 0.03299501 & 0.15499158 & 0.13723551 \\
\hline & $2 s$ & 0.53775806 & 0.52848185 & 1.19331404 & 1.18279881 \\
\hline & $2 p$ & 0.39384940 & 0.38433489 & 0.97498406 & 0.96480121 \\
\hline & $3 s$ & 0.15142686 & 0.13370041 & 0.38944296 & 0.36820744 \\
\hline & $3 p$ & 0.11541419 & 0.09707969 & 0.33224471 & 0.31071264 \\
\hline 0.07 & $3 d$ & 0.10539851 & 0.08820589 & 0.31422331 & 0.29461566 \\
\hline & $4 \mathrm{~s}$ & 0.04461574 & 0.02256958 & 0.14651563 & 0.11634361 \\
\hline & $4 p$ & 0.03210162 & 0.01027558 & 0.12463246 & 0.09412611 \\
\hline & $4 \mathrm{~d}$ & 0.02717185 & 0.00537842 & 0.11590575 & 0.08618019 \\
\hline
\end{tabular}

Figure 2 shows the stabilization plots for Be and $B^{+}$for the unscreened case. The stabilized plateaus (slowly varying energy levels) in the stabilization plots indicate the existence of resonances at the corresponding energies, as indicated by arrows in the stabilization plots. We recognise those resonance states as $3 s n l\left({ }^{1} S\right)$ states. For beryllium, the appearance of one stabilized plateau is very 
clear. This obviously corresponds to the $3 s^{2}\left({ }^{1} S\right)$ state. However, for $B^{+}$, a sequence of stabilized plateaus exists. These correspond to $3 s^{2}, 3 p^{2}, 3 s 4 s\left({ }^{1} S\right)$, etc. The energies and widths of these states were determined by calculating the densities of the states around the avoided crossings followed by fitting the densities to the Lorentzian form (6). The best fittings for the $3 s^{2}\left({ }^{1} S\right)$ states corresponding to Figure 2 are presented in Figure 1. It should be mentioned that in each fitting $\left(R^{2}\right)$ is less than 0.99999. We checked the convergence of the resonance parameters by increasing $N$ in the wave function (7). This is shown in Table 2. Our reported results are correct to four decimal places, and the uncertainty was estimated to be a few parts in the last quoted digit. All other reported results in this paper were obtained by using 372 terms in the wave function (7), and energies were measured relative to the corresponding core energies.

Table 2. Energies (in a.u.) and widths (in a.u.) of the DESs of the Be-like ions for $\lambda=0$ lying above the $1 s^{2} 2 p$ threshold for different $N$ in Equation (7). Energies are measured relative to the energy of the corresponding core.

\begin{tabular}{ccccccc}
\hline & \multicolumn{3}{c}{ Energy } & \multicolumn{3}{c}{ Width } \\
\hline & $\begin{array}{c}N=\mathbf{2 5 2} \\
(\boldsymbol{\omega}=\mathbf{1 2})\end{array}$ & $\begin{array}{c}\boldsymbol{N}=\mathbf{3 0 8} \\
(\boldsymbol{\omega}=\mathbf{1 3})\end{array}$ & $\begin{array}{c}\boldsymbol{(}=\mathbf{\omega 7 2} \\
\mathbf{\omega 1 4})\end{array}$ & $\begin{array}{c}\boldsymbol{N}=\mathbf{2 5 2} \\
(\boldsymbol{\omega}=\mathbf{1 2})\end{array}$ & $\begin{array}{c}\boldsymbol{N}=\mathbf{3 0 8} \\
(\boldsymbol{\omega}=\mathbf{1 3})\end{array}$ & $\begin{array}{c}\boldsymbol{n}=\mathbf{3 7 2} \\
\boldsymbol{\omega}=\mathbf{1 4})\end{array}$ \\
\hline $\mathrm{Be}\left(3 s^{2}{ }^{1} S\right)$ & -0.40998 & -0.40989 & -0.40990 & 0.0027581 & 0.0026238 & 0.002602 \\
$B^{+}\left(3 s^{2}{ }^{1} S\right)$ & -0.96376 & -0.96379 & -0.96374 & 0.0019519 & 0.0019713 & 0.001988 \\
\hline
\end{tabular}

In Table 3 we put up our present results for the unscreened case along with the results of some other calculations. It is seen that our present results fairly agree with the results of other calculations $[15,26,27]$. It is to be mentioned that in case of Be we have been able to determine the resonance parameters for $3 s^{2}\left({ }^{1} S\right)$ state only. Higher states, such as $3 p^{2}, 3 s 4 s\left({ }^{1} S\right)$, seem to have been mixed together, and they are not easily to be determined. Similarly, in case of $B^{+}$, we have able to extract resonance parameters for only four states $-3 s^{2}, 3 p^{2}, 3 s 4 s, 3 s 5 s\left({ }^{1} s\right)$ states. It seems that we need to extend the wave function (7) to a great extent for being able to determine the resonance parameters of those states. Superscripts (1), (2), (3), (4) over $E_{r}$ (and $\Gamma$ ) have been used to denote the energy (and width) of $3 s^{2}, 3 p^{2}, 3 s 4 s, 3 s 5 s\left({ }^{1} S\right)$ states of $B^{+}$.

Table 3. Energies (in a.u.) and widths (in a.u.) of the doubly-excited states (DESs) of Be-like ions for $\lambda=0$ lying above the $1 s^{2} 2 p$ threshold. Energies were measured relative to the energy of the corresponding core. Rows (a), (b) and (c) correspond to the results of Refs. [16,26,27], respectively.

\begin{tabular}{|c|c|c|c|c|c|c|c|c|c|c|}
\hline & \multicolumn{2}{|c|}{ Be } & \multicolumn{8}{|c|}{$B^{+}$} \\
\hline & $-E_{r}$ & $\Gamma$ & $-E_{r}^{(1)}$ & $\Gamma^{(1)}$ & $-E_{r}^{(2)}$ & $\Gamma^{(2)}$ & $-E_{r}^{(3)}$ & $\Gamma^{(3)}$ & $-E_{r}^{(4)}$ & $\Gamma^{(4)}$ \\
\hline Present & 0.40990 & 0.00260 & 0.96374 & 0.00199 & 0.82887 & 0.01347 & 0.75457 & 0.00106 & 0.69710 & 0.00094 \\
\hline (a) & 0.40817 & 0.00295 & & & & & & & & \\
\hline (b) & 0.40891 & 0.00301 & & & & & & & & \\
\hline (c) & 0.4105 & 0.00365 & 0.9638 & 0.00312 & 0.8246 & 0.01301 & 0.7541 & 0.00156 & 0.6974 & 0.00119 \\
\hline
\end{tabular}

The behaviour of the resonance energy with varying screening parameters is presented in Figure 3. The numerical results of energy and width are shown in Table 4 . It was observed that energy increases with increasing $\lambda$, and ultimately, the corresponding state disappears as the energy approaches the $3 S$ state of the corresponding one-electron ion. The resonance states are sustained in SSCP for larger screening parameter values than in ECSCP. This is expected, because with an increasing $\lambda$, the interaction potential becomes weaker. Furthermore, for a given value of $\lambda$, ECSCP is weaker than SSCP. 

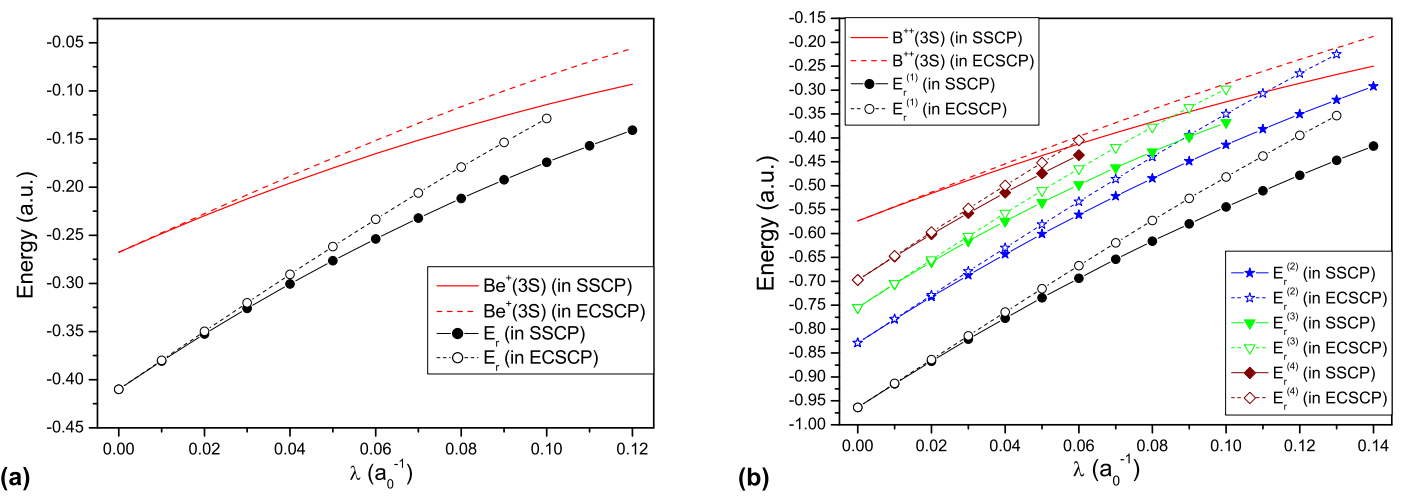

Figure 3. Resonance energies $E_{r}$ as a function of the screening parameter $\lambda$. (a) Be, (b) $B^{+}$.

Table 4. Energies (in a.u.) and widths (in a.u.) of the DESs of the Be-like ions in SSCP and ECSCP lying above the $1 s^{2} 2 p$ threshold for various values of the screening parameter $(\lambda)$ (in $a_{0}^{-1}$ ). Energies were measured relative to the energy of the corresponding core. Rows corresponding to ${ }^{a}$ and ${ }^{b}$ denote the results for SSCP and ECSCP, respectively.

\begin{tabular}{ccccccccccc}
\hline \multicolumn{1}{c}{ Be } \\
\hline$\lambda$ & $-\boldsymbol{E}_{\boldsymbol{r}}$ & $\boldsymbol{\Gamma}$ & $-\boldsymbol{E}_{\boldsymbol{r}}^{(\mathbf{1})}$ & $\boldsymbol{\Gamma}^{(\mathbf{1})}$ & $-\boldsymbol{E}_{r}^{(\mathbf{2})}$ & $\boldsymbol{\Gamma}^{(\mathbf{2})}$ & $-\boldsymbol{E}_{r}^{(\mathbf{3})}$ & $\boldsymbol{\Gamma}^{(3)}$ & $-\boldsymbol{E}_{r}^{(\mathbf{4})}$ & $\boldsymbol{\Gamma}^{(4)}$ \\
\hline 0.0 & 0.40990 & 0.00260 & 0.96374 & 0.00199 & 0.82887 & 0.01347 & 0.75457 & 0.00106 & 0.69710 & 0.00094 \\
$0.01^{a}$ & 0.38051 & 0.00260 & 0.91447 & 0.00198 & 0.77974 & 0.01343 & 0.70576 & 0.00106 & 0.64822 & 0.00094 \\
$0.01^{b}$ & 0.37981 & 0.00261 & 0.91359 & 0.00199 & 0.77879 & 0.01332 & 0.70449 & 0.00106 & 0.64711 & 0.00094 \\
$0.03^{a}$ & 0.32589 & 0.00254 & 0.82117 & 0.00196 & 0.68692 & 0.01330 & 0.61566 & 0.00104 & 0.55710 & 0.00101 \\
$0.03^{b}$ & 0.32008 & 0.00260 & 0.81380 & 0.00199 & 0.67926 & 0.01330 & 0.60561 & 0.00106 & 0.54811 & 0.00095 \\
$0.05^{a}$ & 0.27654 & 0.00244 & 0.73450 & 0.00193 & 0.60107 & 0.01283 & 0.53475 & 0.00101 & 0.47432 & 0.00116 \\
$0.05^{b}$ & 0.26181 & 0.00255 & 0.71549 & 0.00198 & 0.58136 & 0.01340 & 0.51017 & 0.00105 & 0.45181 & 0.00101 \\
$0.07^{a}$ & 0.23215 & 0.00231 & 0.65407 & 0.00187 & 0.52183 & 0.01214 & 0.46226 & 0.00097 & & \\
$0.07^{b}$ & 0.20596 & 0.00246 & 0.61954 & 0.00195 & 0.48611 & 0.01314 & 0.41994 & 0.00104 & & \\
$0.09^{a}$ & 0.19242 & 0.00216 & 0.57956 & 0.00180 & 0.44878 & 0.01131 & 0.39751 & 0.00095 & & \\
$0.09^{b}$ & 0.15347 & 0.00231 & 0.52673 & 0.00191 & 0.39440 & 0.01266 & 0.33657 & 0.00106 & & \\
$0.10^{a}$ & 0.17422 & 0.00208 & 0.54444 & 0.00176 & 0.41453 & 0.01094 & 0.36787 & 0.00095 & & \\
$0.10^{b}$ & 0.12876 & 0.00223 & 0.48172 & 0.00188 & 0.35014 & 0.01226 & 0.29793 & 0.00113 & & \\
\hline
\end{tabular}

The variation in the width of the DESs with varying $\lambda$ is presented in Figure 4 . We notice that the width of the $3 s^{2}\left({ }^{1} S\right)$ state decreases with an increasing $\lambda$ value. Note that this state lies well below the $B e^{+}(3 s)$ (or $B^{++}(3 s)$ ) and exhibits the properties of Feshbach resonance. The autoionization of Feshbach resonance is usually accomplished through momentum transfer. In a screening environment, slow movements of the electrons prolong the autoionization lifetime, and as a result, the resonance width decreases due to the uncertainty principle. The same thing happens for the $3 p^{2}\left({ }^{1} \mathrm{~S}\right)$ state too, i.e., the width decreases for increasing $\lambda$ values. However, the behaviour of the width for small values of $\lambda$ seems to be yielded by the oscillatory behaviour of ECSCP. Such a phenomenon was also observed in earlier investigations $[1,43,45]$. The $3 s 4 s\left({ }^{1} S\right)$ state is rather close to the threshold of the $\mathrm{Be}^{+}(3 s)$ (or $B^{++}(3 s)$ ) state. As a result, with an increasing $\lambda$ of the wave packet, this state crosses over the threshold and autoionizes through tunneling in a manner similar to that of shape resonance. In general, the width of shape resonance increases as its energy moves away from the threshold. In our case, it is the part of the wave function which crosses over and moves away from the threshold as $\lambda$ is increased. In shape resonance, the potential barrier through which the electrons tunnel out becomes narrower as the resonance energy is separated further from the threshold, and the tunneling time becomes shorter, leading to the broadening of the width, again due to the uncertainty principle. Thus, the simultaneous effect of a decreasing width owing to the Feshbach resonance character and an increasing width owing to the shape resonance controls the behaviour of the width of the $3 s 4 s\left({ }^{1} S\right)$ state such a behavior (first 
decreases and then increases). The same reason can be put up to qualitatively explain the behavior of the $3 s 5 s\left({ }^{1} S\right)$ state. This state is very close to the $B e^{+}(3 s)$ (or $B^{++}(3 s)$ ) threshold, and autoionization is dominated by the tunneling process similar to that for shape resonance. As a result, the width increases with increasing $\lambda$. We should mention here that this is our qualitative explanation. A detailed quantitative study in this regard is, of course, of special interest in the future.
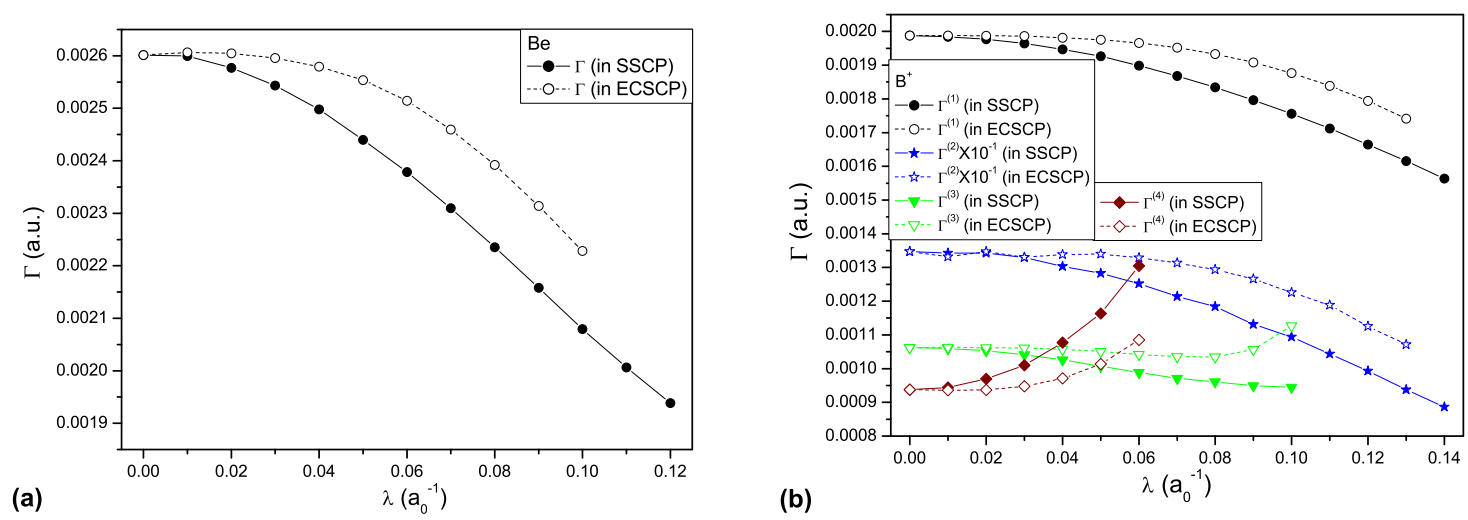

Figure 4. (a) Resonance widths $(\Gamma)$ corresponding to the resonance energies in Figure 3. (a) Be, (b) $B^{+}$.

\section{Conclusions}

The behaviour of the lowest ${ }^{1} S$ DESs of Be-like ions under SCP was investigated by employing the $\mathrm{SM}$. Resorting to the MMP enabled us to evade the cumbersome calculations required for four-electron atomic systems. Though there was an indication of the existence of a series of DESs of Be and $B^{+}$, we were able to compute the energy and width of the $3 s^{2}\left({ }^{1} s\right)$ state of Be and the $3 s^{2}, 3 p^{2}, 3 s 4 s, 3 s 5 s$ $\left({ }^{1} S\right)$ states of $B^{+}$using a 372-term wave function. It seems that higher states of Be are mixed together. The use of a wave function of larger size might resolve the problem. Our present findings show that the energy and width of the DESs undergo significant changes with varying strengths of the screening parameter. In particular, the changes introduced in the width are quite interesting. Though we have given a qualitative explanation behind these changes, it is worth studying these changes quantitatively. We hope that our present findings will be of importance to research in atomic physics, plasma physics, astrophysics, and solid state physics. Moreover, we invite experimental works to validate our present findings.

Author Contributions: The authors have contributed equally to the paper. Both authors have read and approved the final manuscript.

Funding: Y.K.H. was partially funded by the Ministry of Science and Technology in Taiwan, Republic of China, with grant number MOST 104-2112-M-001-032. A.G. was partially funded by the University Grants Commission, New Delhi, India with grant number F. No. 43-415/2014(SR)).

Acknowledgments: We are thankful to reviewers for encouragement and helpful suggestions.

Conflicts of Interest: The authors declare no conflict of interest.

\section{References}

1. Ghoshal, A.; Ho, Y.K. Resonances below the $\operatorname{Ps}(n=2)$ excitation threshold of the $e^{+}-H e\left(1 s 2 s^{3} S^{e}\right)$ system interacting with screened potentials. J. Phys. B At. Mol. Opt. Phys. 2017, 50, 075001. [CrossRef]

2. Janev, R.K.; Zhang, S.; Wang, J. Review of quantum collision dynamics in Debye plasmas. Matter Radiat. Extremes 2016, 1, 237-248. [CrossRef]

3. Saha, J.K.; Mukherjee, T.K.; Mukherjee, P.K.; Fricke, B. Effect of strongly coupled plasma on the doubly excited states of heliumlike ions. Eur. Phys. J. D 2012, 66, 43. [CrossRef]

4. Murillo, M.S.; Weisheit, J.C. Dense plasmas, screened interactions, and atomic ionization. Phys. Rep. 1998, 302, 1-65. [CrossRef] 
5. Weisheit, J.C. Atomic excitation in dense plasmas. Adv. At. Mol. Phys. 1989, 25, 101-131.

6. Salzmann, D. Atomic Physics in Hot Plasmas; Oxford University Press: Oxford, UK, 1998.

7. Scheibner, K.; Weisheit, J.C.; Lane, N.F. Plasma screening effects on proton-impact excitation of positive ions. Phys. Rev. A 1987, 35, 1252. [CrossRef]

8. Nguyen, H.; Koenig, M.; Benredjem, D.; Caby, M.; Coulaud, G. Atomic structure and polarization line shift in dense and hot plasmas. Phys. Rev. A 1986, 33, 1279. [CrossRef]

9. Whitten, B.L.; Lane, N.F.; Weisheit, J.C. Plasma-screening effects on electron-impact excitation of hydrogenic ions in dense plasmas. Phys. Rev. A 1984, 29, 945. [CrossRef]

10. Ichimaru, S. Strongly coupled plasmas: High-density classical plasmas and degenerate electron liquids. Rev. Mod. Phys. 1982, 54, 1017. [CrossRef]

11. Margenau, H.; Lewis, M. Structure of Spectral Lines from Plasmas. Rev. Mod. Phys. 1959, 31, 569. [CrossRef]

12. Shukla, P.K.; Eliasson, B. Screening and wake potentials of a test charge in quantum plasmas. Phys. Lett. A 2008, 372, 2897-2899. [CrossRef]

13. Ghoshal, A.; Ho, Y.K. Ground states of helium in exponential-cosine-screened Coulomb potentials. J. Phys. B At. Mol. Opt. Phys. 2009, 42, 075002. [CrossRef]

14. Hall, R.L. The Yukawa and Hulthen potentials in quantum mechanics. J. Phys. A Math. Gen. 1992, $25,1373$. [CrossRef]

15. Li, K.; Zhuo, L.; Gou, B. Energies, radiative rates, and Auger widths of the doubly-excited $1 s^{2} 3 l 3 l^{\prime}$ states of the Be isoelectronic sequence. J. Quant. Spectrosc. Radiat. Transf. 2016, 182, 94-101. [CrossRef]

16. Zajac, T.; Simulik, V.; Tymchyk, R. The Beryllium Atom Lowest Autoionizing States in the Method of Interacting Configurations in the Complex Number Representation. Int. J. Theor. Math. Phys. 2016, 6, 111-116.

17. Cardona, J.C.; Sanz-Vicario, J.L. Autoionizing states in beryllium-like atomic systems using explicitly correlated coordinates. J. Phys. B At. Mol. Opt. Phys. 2008, 41, 055003. [CrossRef]

18. Roy, U.; Ho, Y.K. Computation of doubly-excited $1 s^{2} 3 l 3 l^{\prime}{ }^{1} P^{0}{ }^{1} F^{0}$ states in beryllium-like ions. Indian J. Phys. 2008, 82, 387.

19. Murakami, I.; Safronova, U.I.; Kato, T. Dielectronic recombination rate coefficients to excited states of Be-like oxygen and dielectronic satellite lines. Can. J. Phys. 2002, 80, 1525-1542. [CrossRef]

20. Pong, W.J.; Ho, Y.K. Doubly-excited states of beryllium-like ions. Phys. Scr. 2000, 61, 690. [CrossRef]

21. Murakami, I.; Safronova, U.I.; Kato, T. Dielectronic recombination rate coefficients to excited states of Ne VII from Ne VIII. J. Phys. B At. Mol. Opt. Phys. 1999, 32, 5331. [CrossRef]

22. Pong, W.J.; Ho, Y.K. Calculations of doubly excited states of beryllium-like ions using the stabilization method. J. Phys. B At. Mol. Opt. Phys. 1998, 31, 2177. [CrossRef]

23. Safronova, U.; Kato, T.; Ohira, M. Dielectronic recombination rate coefficients to the excited states of CIII from CIV. J. Quant. Spectrosc. Radiat. Transf. 1997, 58, 193. [CrossRef]

24. Van der Hart, H.W.; Hansen, J.E. Competition between radiative and non-radiative decay for the doubly-excited $l s^{2} 3 \ln l^{\prime}$ states in $O^{4+}$. J. Phys. B At. Mol. Opt. Phys. 1993, 26, 3297. [CrossRef]

25. Van der Hart, H.W.; Hansen, J.E. Calculations of doubly-excited states in $\mathrm{He}, \mathrm{N}^{5+}$ and $\mathrm{N}^{3+}$ using B-splines. J. Phys. B At. Mol. Opt. Phys. 1992, 25, 41. [CrossRef]

26. Lengyel, V.I.; Navrotsky, V.T.; Sabad, E.P. Resonant scattering of low-energy electrons by $\mathrm{Be}^{+}$and $\mathrm{Mg}^{+}$ions. J. Phys. B At. Mol. Opt. Phys. 1990, 23, 2847. [CrossRef]

27. Bachau, H.; Galan, P. Resonance parameters and properties of Beryllium-like Doubly excited states: $4 \leq Z \leq 10$. At. Data Nucl. Data Tables 1990, 44, 305-348. [CrossRef]

28. Bachau, H.; Galan, P.; Martin, F. Feshbach-model potential approach for the study of resonant and bound states of Be-like ions. Phys. Rev. A 1990, 41, 3534. [CrossRef] [PubMed]

29. Chen, Z.; Lin, C.D. Calculations of energies of intrashell doubly excited states of beryllium-like ions. J. Phys. B At. Mol. Opt. Phys. 1989, 22, 2875. [CrossRef]

30. Vaeck, N.; Hansen, J.E. Calculations for $1 s^{2} 3 l 3 l^{\prime}$ states in $\mathrm{C}^{2+}, \mathrm{N}^{3+}, \mathrm{O}^{4+}, \mathrm{Ne}^{6+}$ and $\mathrm{X} e^{50+}$. J. Phys. B At. Mol. Opt. Phys. 1989, 22, 3137. [CrossRef]

31. Bordenave-Montesquieu, A.; Moretto-Capelle, P.; Bordenave-Montesquieu, D. An accurate test of calculated positions and lifetimes for $N e^{6+}\left(1 s^{2} 3 \ln l^{\prime}\right){ }^{1} L$ states $(n=3$ and 4$)$ using a high-resolution electron spectroscopy. Nucl. Instrum. Meth. Phys. Res. B 2003, 205, 74-77. [CrossRef] 
32. Bordenave-Montesquieu, A.; Moretto-Capelle, P.; Bordenave-Montesquieu, D. High-resolution electron spectroscopy of the $1 s^{2} 3 \ln l^{\prime}$ Be-like series in oxygen and neon. Test of theoretical data: I. Experimental method and theoretical background. J. Phys. B At. Mol. Opt. Phys. 2003, 36, 47. [CrossRef]

33. Bordenave-Montesquieu, A.; Moretto-Capelle, P.; Bordenave-Montesquieu, D. High-resolution electron spectroscopy of the $1 s^{2} 3 \operatorname{lnl} l^{\prime}$ Be-like series in oxygen and neon. Test of theoretical data: II. Experimental results. J. Phys. B At. Mol. Opt. Phys. 2003, 36, 65. [CrossRef]

34. Bordenave-Montesquieu, A.; Moretto-Capelle, P.; Bordenave-Montesquieu, D. Experimental positions and lifetimes of Be-like $1 s^{2} 3 \ln l^{\prime}\left(n=3\right.$ to 5) states of $\mathrm{O}^{4+}$ and $\mathrm{Ne}^{6+}$ ions investigated by high resolution electron spectroscopy: Test of calculations. Phys. Scr. 1999, 80, 372. [CrossRef]

35. Kitazawa, S.; Tanabe, K.; Machida, S.; Matsui, Y.; Ida, H.; Takayanagi, T.; Wakiya, K.; Iemura, K.; Currell, F.; Ohtani, S.; et al. Electron spectra from singlet and triplet states of $N^{3+}\left(1 s^{2} 3 l 3 l^{\prime}\right.$ and $\left.1 s^{2} 3 l 4 l^{\prime}\right)$ produced by low-energy $\mathrm{N}^{5+}+\mathrm{He}$, Ne and Ar collisions. J. Phys. B At. Mol. Opt. Phys. 1998, 31, 3233. [CrossRef]

36. Nakamura, N.; Ida, H.; Matsui, Y.; Wakiya, K.; Takayanagi, T.; Koide, M.; Currell, F.J.; Kitazawa, S.; Suzuki, H.; Ohtani, S.; et al. Electron spectra from singlet and triplet states of $O^{4+}\left(1 s^{2} 3 l 3 l^{\prime}, 1 s^{2} 3 l 4 l^{\prime}\right)$ produced by 60 and $120 \mathrm{keV} \mathrm{O}^{6+}+\mathrm{He}, \mathrm{Ne}$, Ar collisions. J. Phys. B At. Mol. Opt. Phys. 1995, 28, 4743. [CrossRef]

37. Nakamura, N.; Nabeshima, T.; Currell, F.J.; Kanai, Y.; Kitazawa, S.; Koide, M.; Sakaue, H.A.; Ida, H.; Matsui, Y.; Wakiya, K.; et al. Ejected-electron spectra from the triplet states of $O^{4+}\left(1 s^{2} 3 l 3 l^{\prime}\right)$ produced by $\mathrm{O}^{6}+\mathrm{O}_{2}$ collisions. J. Phys. B At. Mol. Opt. Phys. 1994, 27, L785. [CrossRef]

38. Clark, C.W.; Fassett, J.D.; Lucatorto, T.B.; Moore, L.J. Observation of autoionizing states of beryllium by resonance-ionizationmass spectrometry. J. Opt. Soc. Am. B 1985, 2, 891-896. [CrossRef]

39. Cavaliere, P.; Ferrante, G. Model-Potential Theory of Positron-Alkali-Atom Bound States. I: Ground-State Energy and Atomic Properties. Nuovo Cimento 1973, 14, 127-146. [CrossRef]

40. Hilbert, A. Model potentials in atomic structure. Adv. At. Mol. Phys. 1982, 18, 309-340.

41. Laughlin, C.; Victor, G.A. Model-Potential methods. Adv. At. Mol. Phys. 1988, 25, 163-194.

42. Schweizer, W.; Fabbinder, P. Model potentials for alkali metal atoms and li-like ions. At. Data Nucl. Data Tables 1999, 72, 33-55. [CrossRef]

43. Ghoshal, A.; Ho, Y.K. Autoionization states of the positronium negative ion in exponential cosine-screened Coulomb potentials. Eur. Phys. J. D 2010, 56, 151-156. [CrossRef]

44. Ghoshal, A.; Ho, Y.K. Doubly excited resonance states of helium in exponential cosine-screened Coulomb potentials. Phys. Rev. A. 2009, 79, 062514. [CrossRef]

45. Ghoshal, A.; Ho, Y.K. Properties of the Positronium Negative Ion Interacting with Exponential Cosine-Screened Coulomb Potentials. Few-Body Syst. 2009, 46, 249-256. [CrossRef]

46. Fang, T.K.; Ho, Y.K. Determination of resonance energies and widths of $\mathrm{Mg} 3 p n l^{1} D^{e}$ and ${ }^{1} F^{0}$ doubly excited states by the stabilization method with the B-spline-based configuration interaction approach. J. Phys. B At. Mol. Opt. Phys. 1999, 32, 3863. [CrossRef]

47. Tan, S.S.; Ho, Y.K. Determination of Resonance Energy and Width by Calculation of the Density of Reesonance States Using the Stabilisation Method. Chin. J. Phys. 1997, 35, 701-707.

48. Bowman, J.M. Collision lifetime approach to recombination and a new derivation of RRKM theory. J. Phys. Chem. 1986, 90, 3492-3495. [CrossRef]

49. Bashkin, S.; Stoner, J.O. Atomic Energy Levels and Gotrian Diagrams; North-Holland: Amsterdam, The Netherlands, 1975; Volume 1.

50. Chung, K.T. Ionization potential of the lithiumlike $1 s^{2} 2 s$ states from lithium to neon. Phys. Rev. A 1991, $44,5421$. [CrossRef] [PubMed]

51. Kelly, R.L. Atomic and Ionic Spectrum Lines below 2000 Angstrom: Hydrogen through Krypton Part I (H-Cr). J. Phys. Chem. Ref. Data 1987, 16, 1.

(C) 2018 by the authors. Licensee MDPI, Basel, Switzerland. This article is an open access article distributed under the terms and conditions of the Creative Commons Attribution (CC BY) license (http:/ / creativecommons.org/licenses/by/4.0/). 